\title{
Controlling a Humanoid Robot Arm for Grasping and Manipulating a Moving Object in the Presence of Obstacles without Cameras
}

\author{
Ali Chaabaani, Mohamed Sahbi Bellamine, and Moncef Gasmi
}

\begin{abstract}
Many of researchers working on robotic grasping tasks assume a stationary or fixed object, others have focused on dynamic moving objects using cameras to record images of the moving object and then they treated their images to estimate the position to grasp it. This method is quite difficult, requiring a lot of computing, image processing... Hence, it should be sought more simple handling method. Moreover, the majorities of robotic arms available for humanoid applications are complex to control and yet expensive. In this paper, we are going to detail the requirements to manipulating a 7-DoF WAM robotic arm equipped with the Barrett hand to grasp and handle any moving objects in the 3-D environment in the presence of obstacles and without using the cameras. We used the OpenRAVE simulation environment. We use an extension of RRT-JT algorithm that interleaves exploration using a Rapidly-exploring Random Tree with exploitation using Jacobian-based gradient descent to control the 7-DoF WAM robotic arm to avoid the obstacles, track a moving object, and grasp planning. We present results in which a moving mug is tracked, stably grasped with a maximum rate of success in a reasonable time and picked up by the Barret hand to a desired position.
\end{abstract}

Index Terms - Grasping, moving object, trajectory planning, robot hand, obstacles.

\section{INTRODUCTION}

The problem of grasping a moving object in the presence of obstacles with a robotic manipulator has been reported in different works. There have been many studies on grasping motion planning for a manipulator to avoid obstacles [1]-[3]. One may want to apply a method used for mobile robots, but it would cause a problem since it only focuses on grasping motion of robot hands and since the configuration space dimension is too large. Motion planning for a manipulator to avoid obstacles, however, which takes account of the interference between machine joints and obstacles, has been extensively studied in recent years and now has reached a practical level. Grasping operations in an environment with obstacles are now commonly conducted in industrial applications and by service robots.

In the field of robotics, many applications have been tailored towards servoing using visual information. The goal is to use information obtained from vision inside a servo loop to control a mobile manipulator [4], [5], [6]. These challenges are the major reason for a limited performance in the tracking and grasping process which can be solved via use of

Manuscript received August 15, 2014; revised November 12, 2014

The authors are with the National Institute of Applied Sciences and Technology (INSAT), Tunisia (e-mail: chaabani.ali@gmail.com, aroussia@insat.rnu.tn, mcfgsm@yahoo.fr). predictive algorithms. [7] developed a system to grasp moving targets using a static camera and precalibrated camera-manipulator transform. [8] proposed a control theory approach for grasping using visual information. [9] presented a system to track and grasp an electric toy train moving in an oval path using calibrated static stereo cameras. [10] proposed a method to grasp efficiently the objects and developed a system able to grasp industrial parts moving on a conveyor belt by controlling a 6DOF robot arm with a camera mounted on its gripper. [11] implemented a real time vision system with a single camera for identifying and intercepting several objects. [12] proposed a visual servo system for real-time tracking and grasping of a moving object and a parallel method was adopted to raise matching speed.

These researchers have recognized that the main problems in the visual servoing are to solve the delay introduced by image processing or the response of the robot system and resolve the target occlusion. These troubles are the major reason for a limited performance in the tracking and grasping process which can be solved through of the use of predictive algorithms. [13] use a prediction module which consists of a linear predictor with the purpose of predicting the location that a moving object will have and thus generate the control signal to move the eyes of a humanoid robot, which is capable of using behavior models similar to those of human infants to track objects. [14] present a tracking algorithm based on a linear prediction of second order solved by the Maximum Entropy Method. It attempts to predict the centroid of the moving object in the next frame, based on several past centroid measurements. [15] represent the tracked object as a constellation of spatially localized linear predictors which are trained on a single image sequence. In a learning stage, sets of pixels whose intensities allow for optimal prediction of the transformations are selected as a support for the linear predictor. [16] presents a binocular eye-to-hand visual servoing system that is able to track and grasp a moving object in real time. In the tracking module, they use three linear predictors (one for each component of the three dimensions) to predict and generate the trajectory that will describe the $3 \mathrm{D}$ object position in the near future, therefore, their manipulator robot is able to track and grasp a moving object, even if the object is temporarily occluded. [17] Implementation of tracking and capturing a moving object using a mobile robot.

The researchers who use the visual servoing system and the cameras for grasping moving object find many difficulties to record images, to treat them, because of a lot computing and image processing and also who use the predictive algorithms find a problem in the complexity of 
algorithms witch based on many calculated and estimation [18]. In this research we want to grasp a moving object with limited motion velocity. This can be done by determining desired position for the object, the robot moves and aligns the end effector with the object and reaches towards it. This paper presents a motion planning and controlling an arm of a humanoid robot for grasping and manipulating of a moving object without cameras. We used an algorithm to control the end effect or pose (position and orientation) with respect to the pose of objects which can be moved in the workspace of the robot. The proposed algorithm successfully grasped a moving object in a reasonable time.

This paper has six main Sections. Section II is devoted to the detailed description of the rapidly-exploring random trees (RRT), and the transpose of the Jacobian is briefly given in Section III. The next Section contains a description of the $\mathrm{WAM}^{\mathrm{TM}}$ arm. In Section V, some results are given. Section VI presents conclusions drawn from this work.

\section{RAPIDLY-EXPLORING RANDOM TREES (RRT)}

In previous work [19], [20], researchers have tackled the motion planning problem by sampling some number of end effector poses from the goal regions and using inverse kinematics(IK) to find joint configurations which place the end effector at the sampled locations. These configurations are then set as goals for a randomized planner, such as an RRT or BiRRT [21], [22]. While often capable of solving the problem at hand, this approach is neither probabilistically complete nor efficient. The issue is that some numbers of samples from the goal regions are chosen a priori as goal configurations, and the planner is forced to use only these goals.

Another approach to planning with certain types of workspace goals is to explore the Configuration space (C-space (see Fig. 1) of the robot with a single search tree that uses heuristics to bias the exploration toward a goal region [23]. However, the goal regions and heuristics defined in [24] are highly problem specific and difficult to tune. Drumwright and Ng-Thow-Hing [25] employ a similar strategy of extending toward a randomly-generated IK solution for a workspace point. In [26], Vande Weghe et al. present the RRT-JT algorithm, which uses a forward-searching tree to explore the $\mathrm{C}$-space and a gradient-descent heuristic based on the Jacobian-transpose to bias the tree toward a work-space goal point.

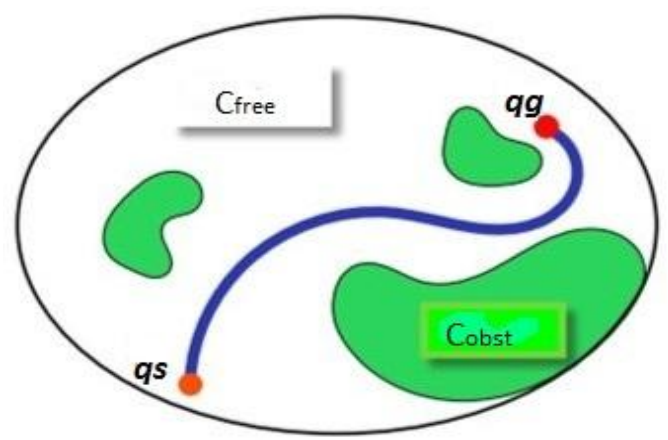

Fig. 1. Configuration space(C-space).

Ref. [27] present two probabilistically complete planners: an extension of RRT-JT, and a new algorithm called
IKBiRRT. Both algorithms function by interleaving exploration of the robot's C-space with exploitation of WGRs(Workspace Goal Regions). The extended RRT-JT (Fig. 2) is designed for robots that do not have such algorithms and is able to combine the configuration space exploration of RRTs with a workspace goal bias to produce direct paths through complex environments extremely efficiently, without the need for any inverse kinematics.

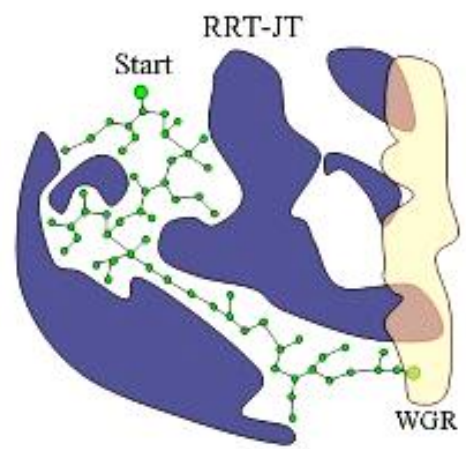

Fig. 2. Depiction of the RRT-JT algorithm searching in C-space: from the start configuration to (WGRs). The blue regions are obstacles, the forward-searching tree is shown with green nodes [18]

\section{USING THE JACOBIAN}

Given a robot arm configuration $q \in Q$ (the configuration space) and a desired end effector goal $x_{g} \in X$, where $X$ is the space of end effector positions R3, we are interested in computing an extension in configuration space from $q$ to wards $x g$. Unfortunately, the mapping from $Q$ to $\mathrm{X}$ is usually nonlinear and very expensive to compute. However, its derivative, called the Jacobian, is a linear map from the tangent space of $Q$ to that of $X$, is expressed as $\boldsymbol{J} \dot{q}=\dot{\mathrm{x}}$, where $x \in X$ is the end effector position (or pose) corresponding to $q$, and can be computed quickly. Ideally, to drive the end effector to a desired configuration $x_{g},\left(d x_{g} / d t \approx 0\right.$ : object moves slowly) we could compute the error $e(t)=\left(x_{g}-x\right)$ and run a controller of the form $\dot{q}=K J^{-1} e$, where $\mathrm{K}$ is a positive gain. In the absence of any obstacles, internal collisions, or joint limits, this simple controller is guaranteed to reach the goal. Unfortunately, in the absence of a closed form solution, the computation of the inverse of the Jacobian must be done numerically at each time step. An alternate approach, is to use the transpose of the Jacobian instead of the inverse. This results in a control law of the form $\dot{q}=K \boldsymbol{J}^{T} e$. The controller eliminates the large overhead of computing the inverse by using the easy-to-compute Jacobian instead. It is easy to show that, under the same obstacle-free requirements as the Jacobian inverse controller, the Jacobian transpose (JT) controller is also guaranteed to reach the goal. The instantaneous motion of the end effector is given by $\dot{x}=\boldsymbol{J} q$ $=\boldsymbol{J}\left(K \boldsymbol{J}^{T} e\right)$. The inner product of this Instantaneous motion with the error vector is given by $e^{T} \dot{x}=k e^{T} J J^{T} e \geq 0$. Since this is always positive, under our assumptions about obstacles, the controller is guaranteed to make forward progress towards the goal [28].

\section{THE WAMTM ARM}

The WAM Arm is a highly dexterous back drivable 
manipulator. It is the only commercially available robotic arm with direct-drive capability supported by Transparent Dynamics between the motors and joints, so its joint-torque control is unmatched and guaranteed stable. It is built to outperform today's conventional robots by offering extra ordinary dexterity, zero backlash, and near- zero friction. The WAM Arm is available in 3 main configurations, 4-DOF, 7DOF, both with human-like kinematics, and 4-DOF with 3-DOF Gimbals. The joint ranges exceed those for conventional robotic arms [29].

We use WAM 7-DOF Arm with attached Barrett Hand.

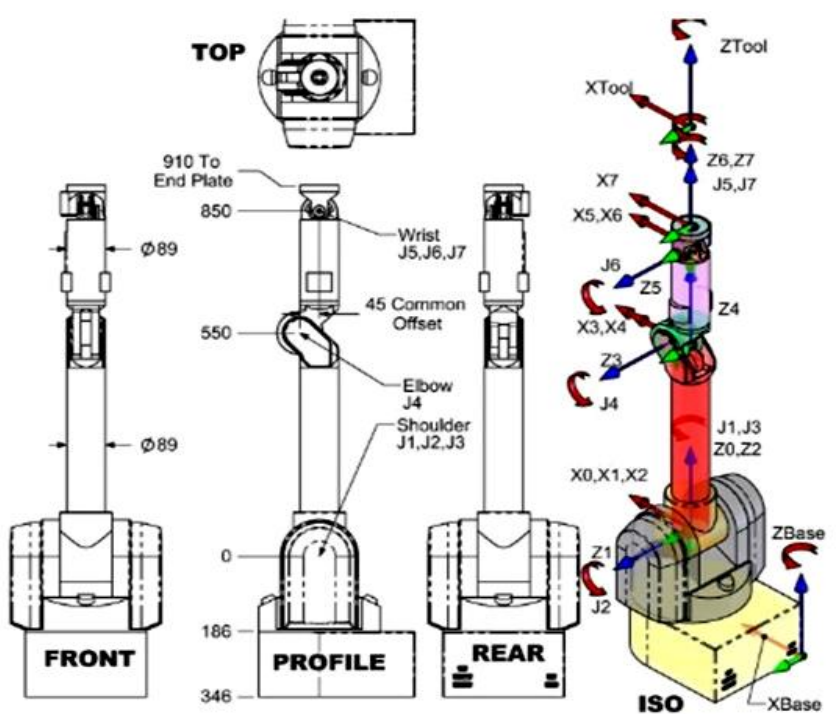

Fig. 3. WAM 7-DOF dimensions and D-H frames [30].

Fig. 3 shows the entire 7-DOF WAM system in the zero position. A positive joint motion is based on the right hand rule for each axis. The following equation of homogeneous transformation in Fig. 4 is used to determine the transformation between the axes $\mathrm{K}$ and $\mathrm{K}-1$.

$$
{ }^{k-1} T_{k}=\left[\begin{array}{cccc}
\cos \theta_{k} & -\sin \theta_{k} \cos \alpha_{k} & \sin \theta_{k} \sin \alpha_{k} & a_{k} \cos \theta_{k} \\
\sin \theta_{k} & \cos \theta_{k} \cos \alpha_{k} & -\cos \theta_{k} \sin \alpha_{k} & a_{k} \sin \theta_{k} \\
0 & \sin \alpha_{k} & \cos \alpha_{k} & d_{K} \\
0 & 0 & 0 & 1
\end{array}\right]
$$

Fig. 4. D-H generalized transform matrix.

- $a_{k-1}=$ the distance from $Z_{k-1}$ to $Z_{k}$ measured along $X_{k-1}$ - $d_{k}=$ the distance from $X_{k-1}$ to $X_{k}$ measured along $Z_{k}$

- $\alpha_{k-1}=$ angle between $Z_{k-1}$ to $Z_{k}$ was approximately $X_{k-1}$ - $\theta_{k}=$ angle between $X_{k-1}$ to $X_{k}$ was approximately $Z_{k}$

The Table I contains the parameters of the arm with 7-DoF.

TABLE I: 7-DOF WAM FRAME PARAMETERS

\begin{tabular}{ccccc}
\hline \hline$K$ & $a_{k}$ & $\alpha_{k}$ & $d_{k}$ & $\theta_{k}$ \\
\hline 1 & 0 & $-\pi / 2$ & 0 & $\theta_{1}$ \\
2 & 0 & $\pi / 2$ & 0 & $\theta_{2}$ \\
3 & 0.045 & $-\pi / 2$ & 0.55 & $\theta_{3}$ \\
4 & -0.045 & $\pi / 2$ & 0 & $\theta_{4}$ \\
5 & 0 & $-\pi / 2$ & 0.3 & $\theta_{5}$ \\
6 & 0 & $\pi / 2$ & 0 & $\theta_{6}$ \\
7 & 0 & 0 & 0.060 & $\theta_{7}$ \\
$\mathrm{~T}$ & 0 & 0 & 0 & \\
\hline \hline
\end{tabular}

As with the previous example, we define the ${ }^{7} \mathrm{~T}_{\text {Tool }}$ frame for our specific end effector. The forward kinematics are determined for any frame on the robot by multiplying all of the transforms up to and including the final frame. To determine the end tip location and orientation we use the following equation:

$$
{ }^{0} T_{\text {Tool }}={ }^{0} T_{1}^{1} T_{2}^{2} T_{3}^{3} T_{4}^{4} T_{5}^{5} T_{6}^{6} T_{7}^{7} T_{T o o l}
$$

\section{RESULTS AND ANALYSIS}

To demonstrate and illustrate the proposed procedure, we present an example which the robot is equipped with a 7-DoF arm (see Fig. 3) and a three-fingered Barrett hand(in fact in each time there are three tests: test 1 , test 2 and test 3 ). The goal is to follow a moving model mug, stably holding it, pick it up and move it to the desired position while avoiding the existing obstacles. The mug was moving in a straight line trajectory in the space with velocity range $8-32 \mathrm{~mm} / \mathrm{s}$. The initial positions of the end effector were $(-0.730 \mathrm{~m}, 0.140 \mathrm{~m}$, $2.168 \mathrm{~m})$ and those of the moving object were $(-0.005 \mathrm{~m},-0.200 \mathrm{~m}, 1.105 \mathrm{~m})$. In order to grasp the moving object stably and move it, the robot hand reaches the object than it closes its fingers.

\section{A. Case Study №1: Moving Object with Velocity $V 1=8 \mathrm{~mm} / \mathrm{s}$ in the Presence of One Obstacle}

As shown in the image sequence in Fig. 5, the tracking and the grasping of the item is achieved efficiently. Fig. 5(a) shows that the hand of the robot keeps a distance from it, the Barret hand and the object are in the initial position, Fig. 5(b) the object moves with the velocity $V 1=8 \mathrm{~mm} / \mathrm{s}$ and the robot moves to the position of the object's centroid, avoids the obstacle, opens the fingers, closes them back and finally grasps it. In Fig. 5(c) the robot picks it up while avoiding obstacle and takes it to a determined position.

To capture a moving object safety without collision and to lift it up stably without slippage, the end effector needs to be controlled while considering the relation between its position, the moving object's position and the obstacle one's. It determines the position of the moving object and of the obstacle (in the middle between the object and the end effector) and select the shortest distance from its current position, while avoiding obstacle in the environment.

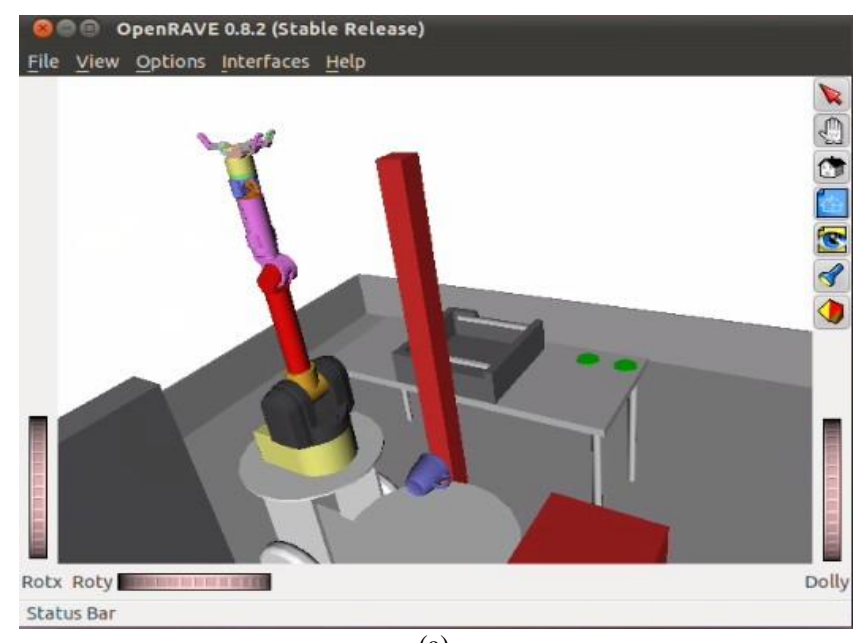

(a) 


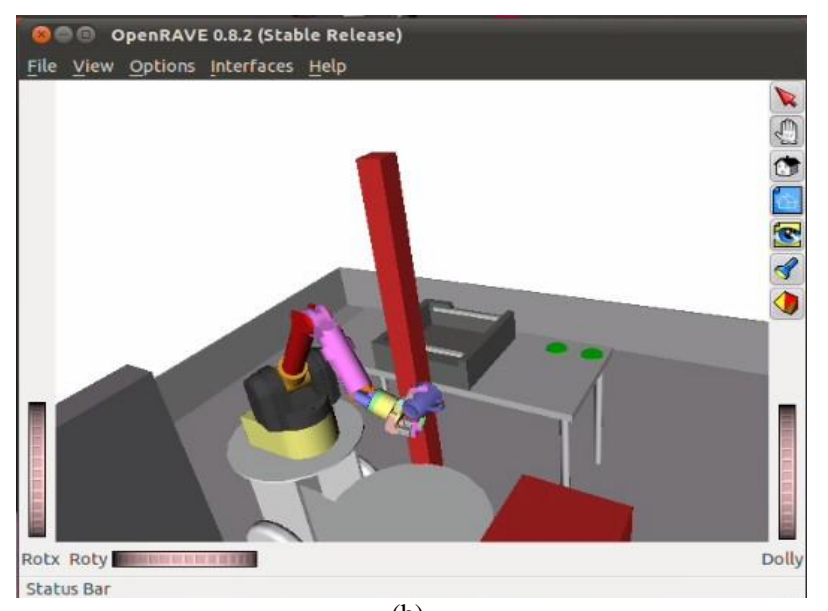

(b)

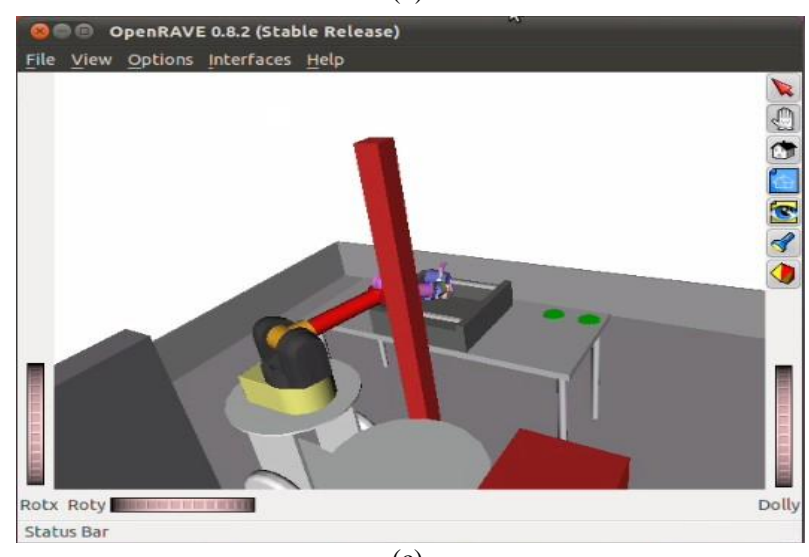

(c)

Fig. 5. Successful grasping of a moving object while avoiding obstacle.

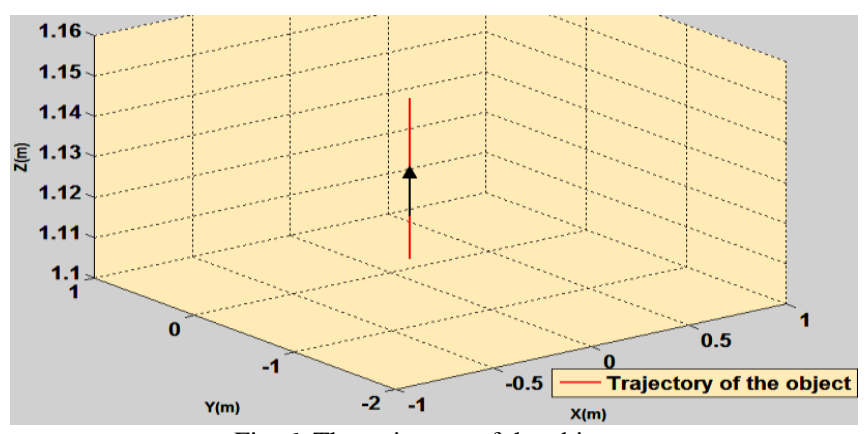

Fig. 6. The trajectory of the object.

Fig. 6 illustrates the trajectory based on the $\mathrm{Z}$ axis, the object moves in a straight line.

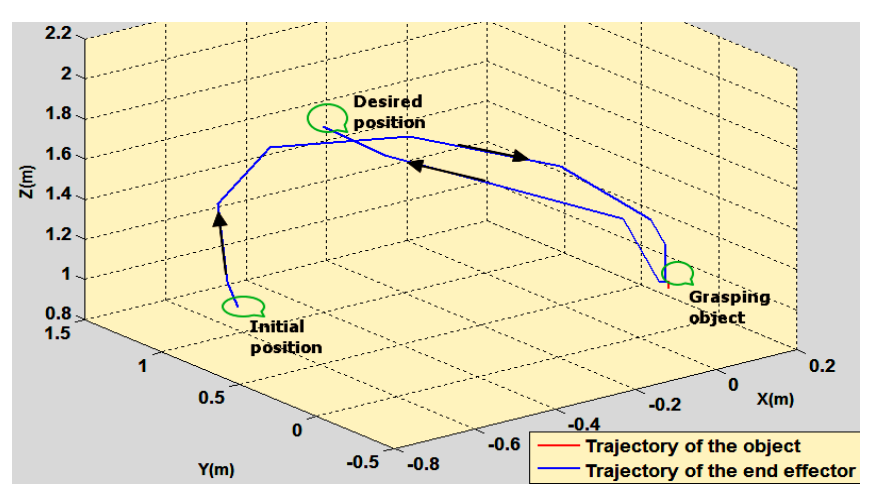

Fig. 7. The trajectory of the end effector and the object.

Fig. 7 represents the curves of the first test: the robot grasps the object in time $T_{\text {grasp }}=2.45 \mathrm{~s}$, which moves according to the $\mathrm{Z}$ axis with velocity $V 1$.

The Table II presents the results of the time for grasping the moving object while avoiding obstacle, and the time to move it to the desired position, as always it moves with velocity $V 1$. Times are nigh in all tests. The direction of the object's movement affects on the time grasping ( $\left.T_{\text {grasp }}\right)$ and on the time to move it to desired position $\left(\mathrm{T}_{\mathrm{end}}\right)$.

TABLE II: OBJect Moves WITH $V_{1}$ IN THE PRESENCE OF OBSTACLE

\begin{tabular}{clllllll}
\hline \hline & \multicolumn{3}{c}{ according $(\mathrm{Z})$ axis } & \multicolumn{3}{c}{ in $(\mathrm{Y}, \mathrm{Z})$} & \multicolumn{2}{c}{$\operatorname{in}(\mathrm{X}, \mathrm{Y}, \mathrm{Z})$} \\
\hline & $\mathrm{T}_{\text {grasp }}(\mathrm{s})$ & $\mathrm{T}_{\text {end }}(\mathrm{s})$ & $\mathrm{T}_{\mathrm{g}_{\text {rasp }}(\mathrm{s})}$ & $\mathrm{T}_{\text {end }}(\mathrm{s})$ & $\mathrm{T}_{\text {grasp }}(\mathrm{s})$ & $\mathrm{T}_{\text {end }}(\mathrm{s})$ \\
\hline test $_{1}$ & 2.45 & 8.91 & 3.11 & 7.63 & 5.16 & 11.38 \\
test $_{2}$ & 2.95 & 9.08 & 2.83 & 9.33 & 2.91 & 7.07 \\
test $_{3}$ & 3.18 & 9.74 & 3.03 & 7.6 & 4.24 & 8.77 \\
\hline \hline
\end{tabular}

B. Case Study №2: Moving Object with Velocity V2=4V1 in the Presence of One Obstacle

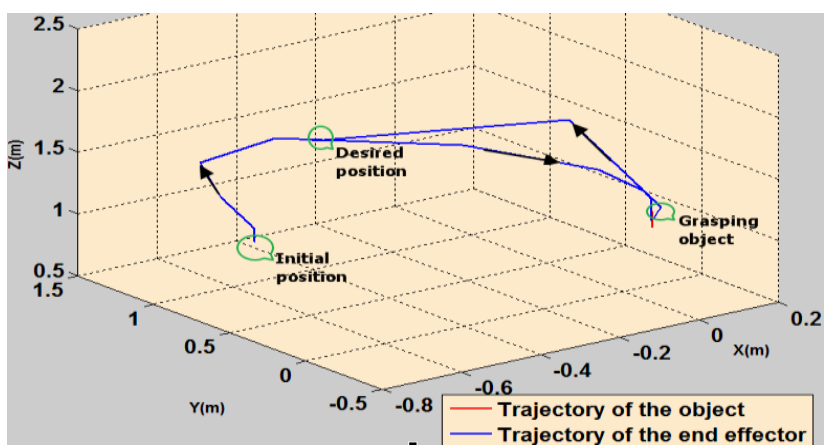

(a)

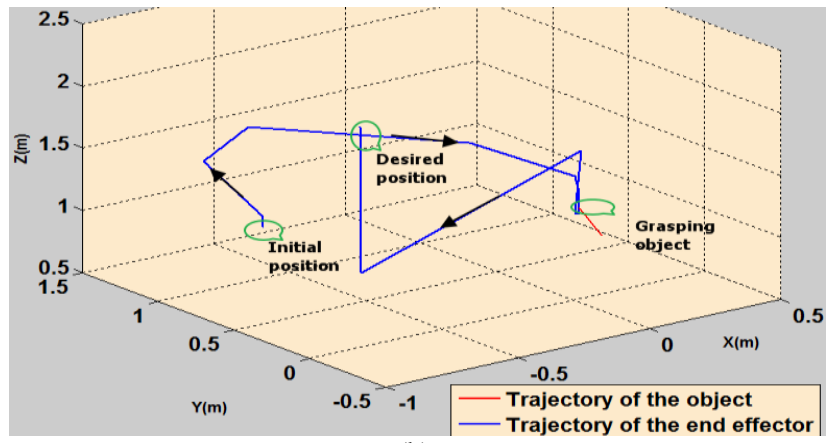

(b)

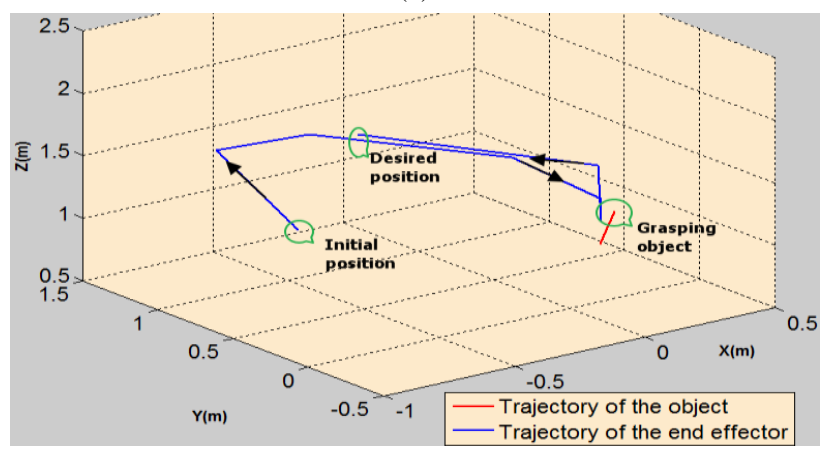

(c)

Fig. 8. The trajectory of the end effector and the object.

Fig. 8(a) illustrates the curves of the second test: the robot grasps the object in time $T_{\text {grasp }}=2.43 \mathrm{~s}$, which moves according to the $\mathrm{Z}$ axis with velocity, Fig. 8(b) represents the curves of the second test: the robot grasps the object in time $\mathrm{T}_{\text {grasp }}=2.74 \mathrm{~s}$, the object moves in the plane $(\mathrm{Y}, \mathrm{Z})$ with velocity $V 2$, in Fig. 8(c) the curves of the second test: the robot grasps the object in time $\mathrm{T}_{\text {grasp }}=2.42 \mathrm{~s}$, the movement is in $\operatorname{space}(\mathrm{X}, \mathrm{Y}, \mathrm{Z})$ with velocity $V 2$. 
TABLE III: OBJect Moves WITH $V_{2}$ IN THE PRESENCE OF OBSTACLE

\begin{tabular}{|c|c|c|c|c|c|c|}
\hline & \multicolumn{2}{|c|}{ according (Z)axis } & \multicolumn{3}{|c|}{$\operatorname{in}(Y, Z)$} & $\operatorname{in}(X, Y, Z)$ \\
\hline & $\mathrm{T}_{\text {grasp }}$ & $\mathrm{T}_{\text {end }}(\mathrm{s})$ & $\mathrm{T}_{\mathrm{g}_{\text {rasp }}}(\mathrm{s})$ & $\mathrm{T}_{\text {end }}$ & $\mathrm{T}_{\text {grasp }}$ & $\mathrm{s} \mathrm{T}_{\text {end }}(\mathrm{s})$ \\
\hline test $_{1}$ & 2.96 & 9.47 & 3.58 & 9.57 & 3 & 9.51 \\
\hline test $_{2}$ & 2.43 & 8.18 & 2.74 & 7.6 & 2.42 & 7.16 \\
\hline test $_{3}$ & 2.37 & 6.87 & 2.63 & 8.13 & 2.54 & 7.35 \\
\hline
\end{tabular}

The Table III shows the results of the time for grasping the moving object which moves with velocity $V 2=4 V 1$ while avoiding obstacle and the time to move the object to the desired position.

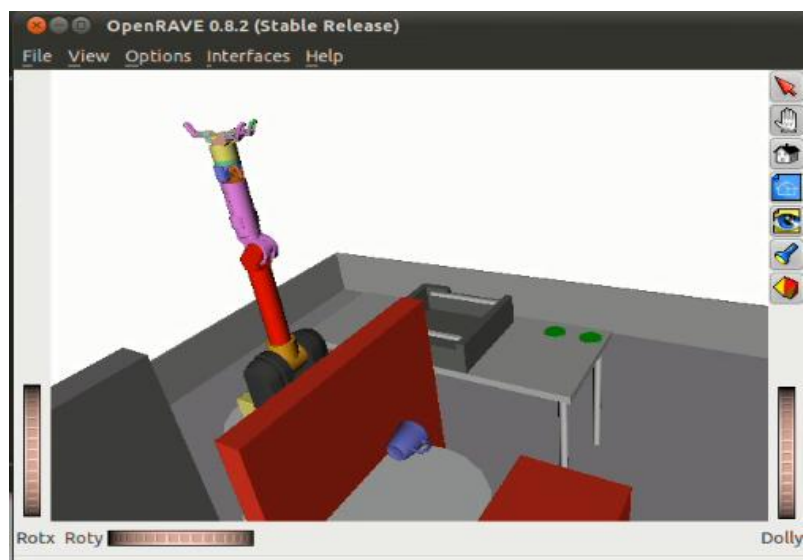

(a)

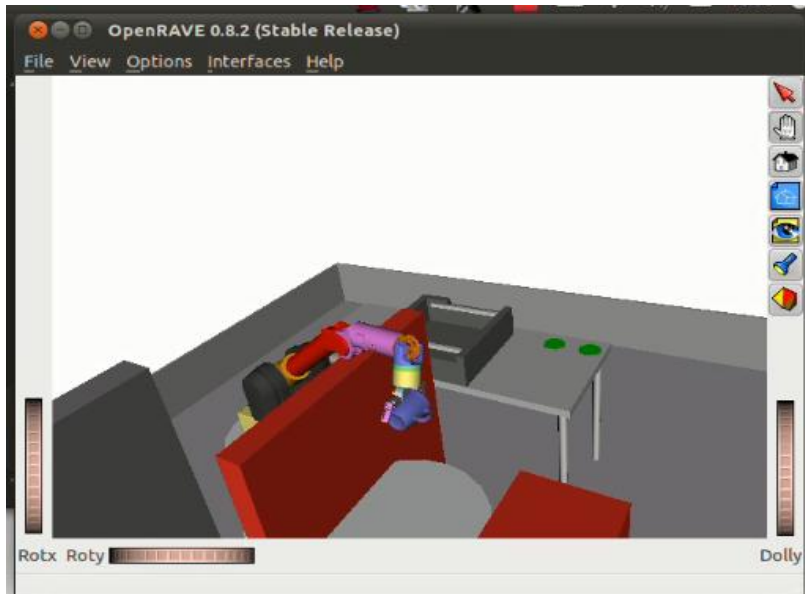

(b)

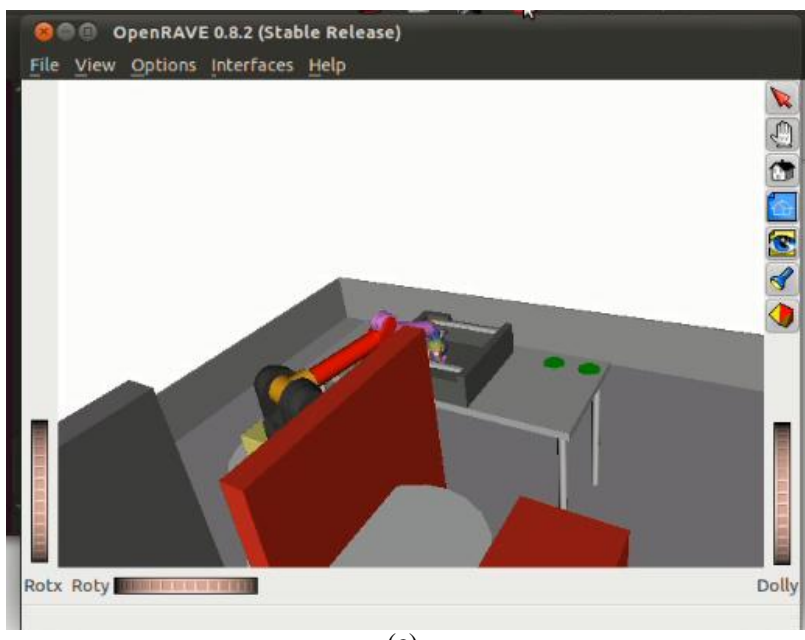

(c)

Fig. 9. The obstacle between the arm and the object.

If we increase the velocity of the object, we see that the results are close but slightly higher. Therefore, increasing the speed affects on the time of grasping the moving object, even the direction of the object's movement affects on the time of holding, we note that in the presence of obstacles the times are slightly higher than in their absence.

As shown in the tables, our algorithm successfully picked it up $100 \%$ of the time, and our robot successfully grasps the objects. We demonstrate that the robot is able to grasp a moving object in a reasonable time. The times recorded in the presence of the obstacle are slightly higher than recorded in the absence of the obstacle.

Fig. 9 illustrates the presence of an obstacle between the arm and the object affects the grasping time of the moving target: $\mathrm{T}_{\mathrm{end}}=8.82 \mathrm{~s}$.

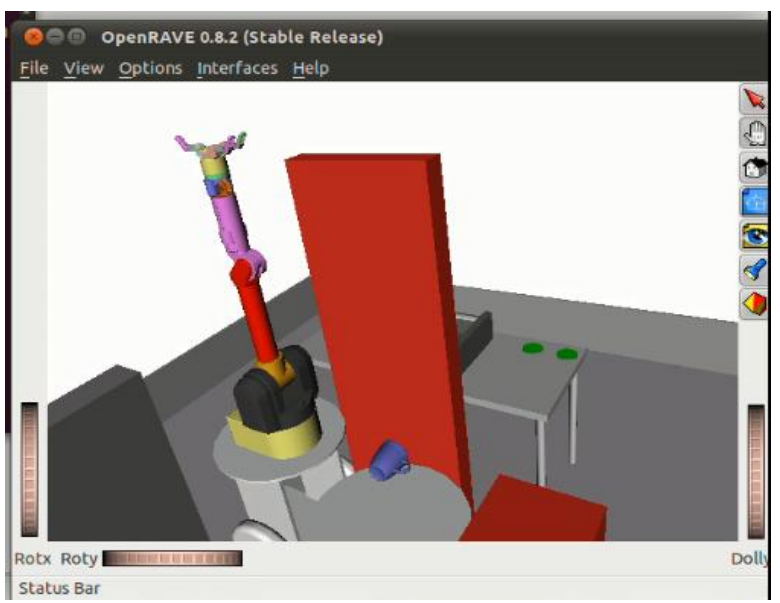

(a)

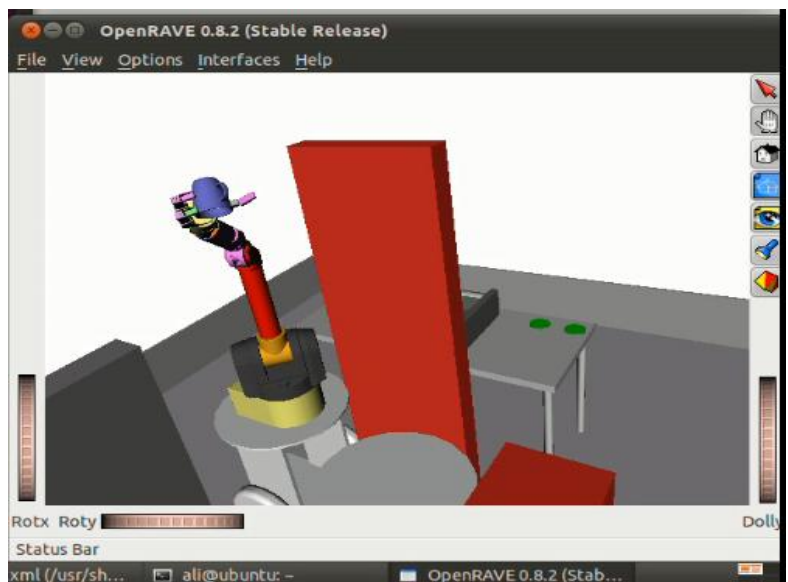

(b)

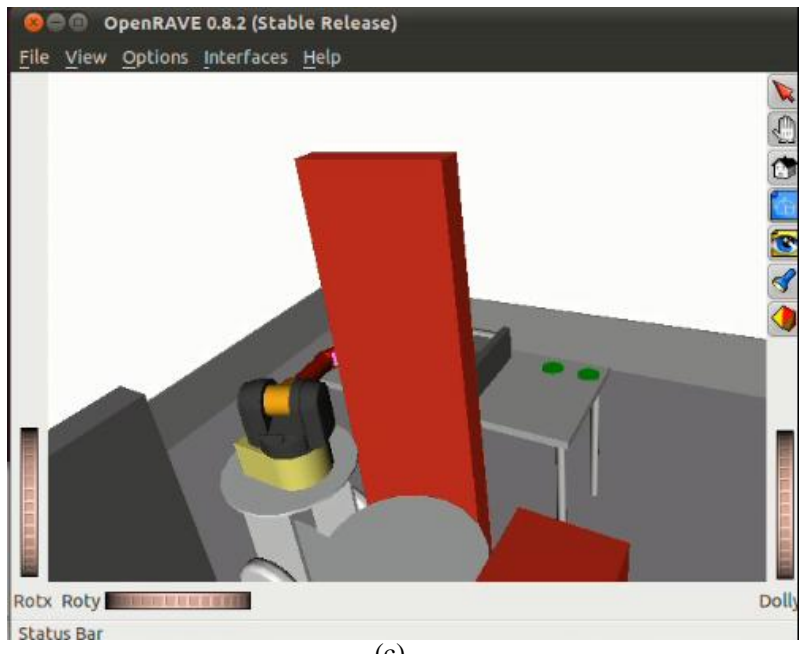

(c)

Fig. 10. The obstacle between the object and the desired position. 
Fig. 10 proves that the presence of an obstacle between the object and the desired position affects on the time to move it to the wanted station: $\mathrm{T}_{\mathrm{end}}=7.45 \mathrm{~s}$.

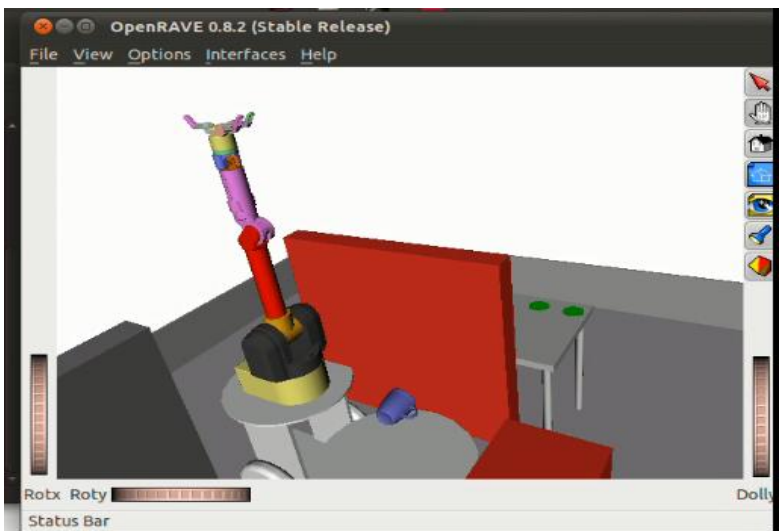

(a)

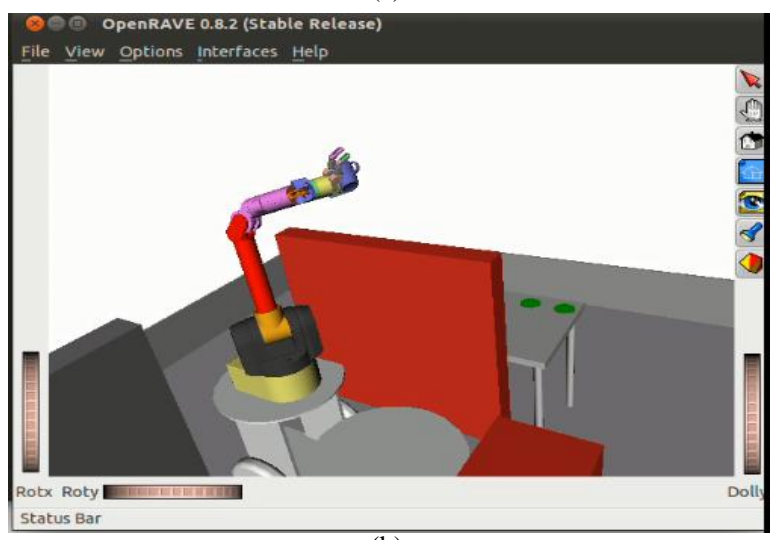

(b)

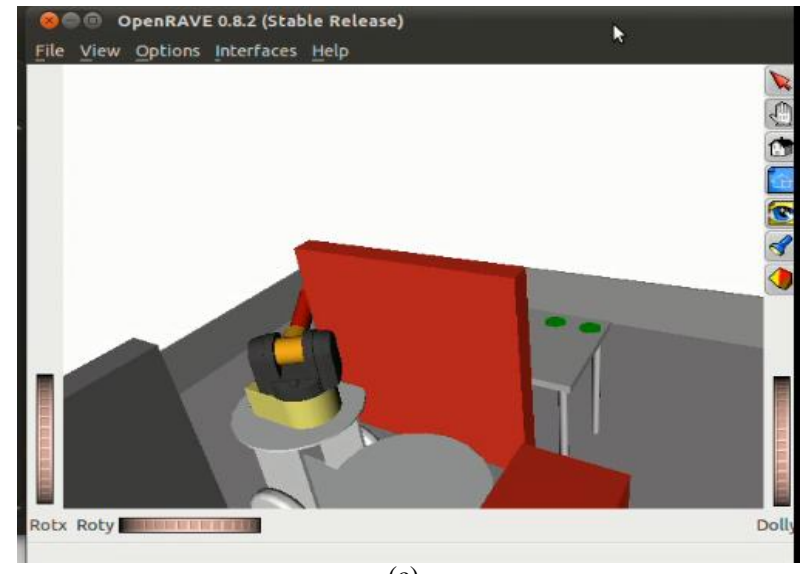

(c)

Fig. 11. The obstacle between the object and the desired position.

The presence of an obstacle between the object and the desired position (see Fig. 11) affects on the time to move the object to the desired position: $\mathrm{T}_{\mathrm{end}}=6.55 \mathrm{~s}$. The height and width of obstacle influence on the end time.

\section{Grasping Object in the Presence of Two Obstacles}

In the presence of obstacles, we plan a path in 7-DoF configuration space that takes the end effector from the starting position to a goal position, avoiding obstacles. For computing the goal orientation of the end effector and the configuration of the fingers, we used a criterion that attempts to minimize the opening of the hand without touching the object being grasped or other nearby obstacles. Finally finds a shortest path from the starting position to possible target positions.
1) Case study №1: Moving object in the presence of two obstacles between the object and the desired position

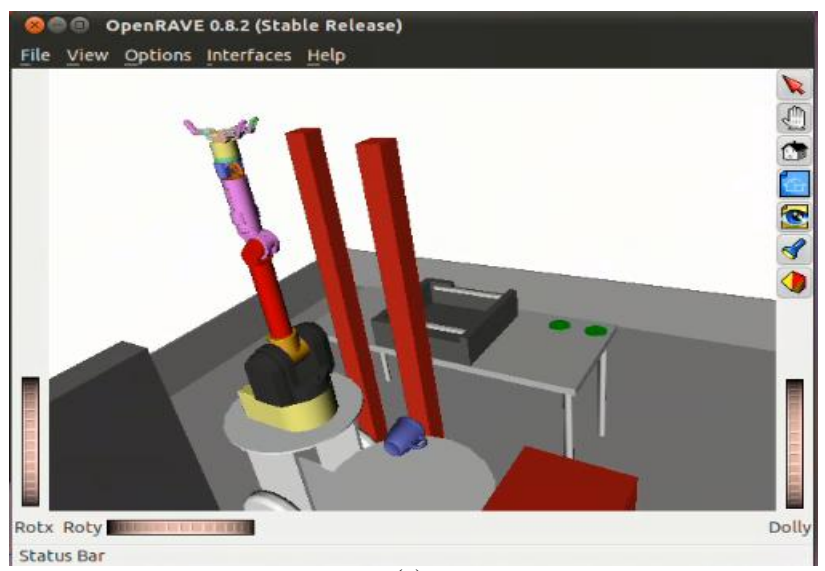

(a)

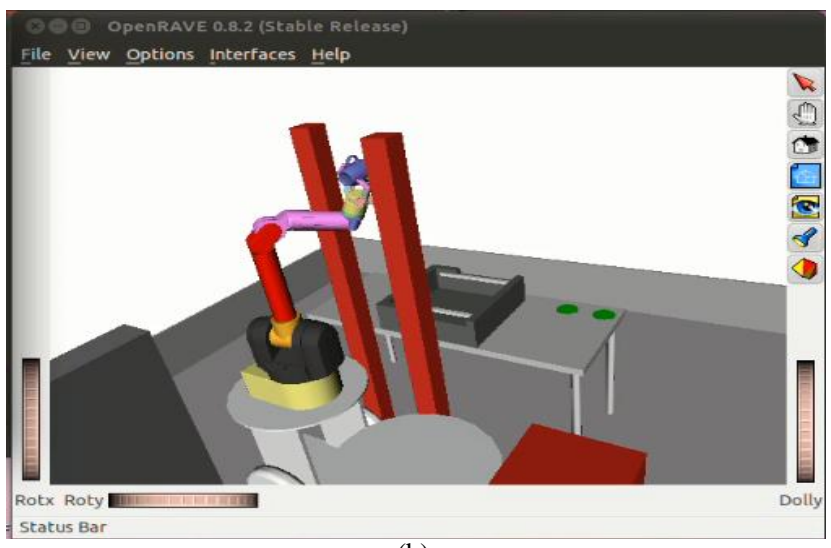

(b)

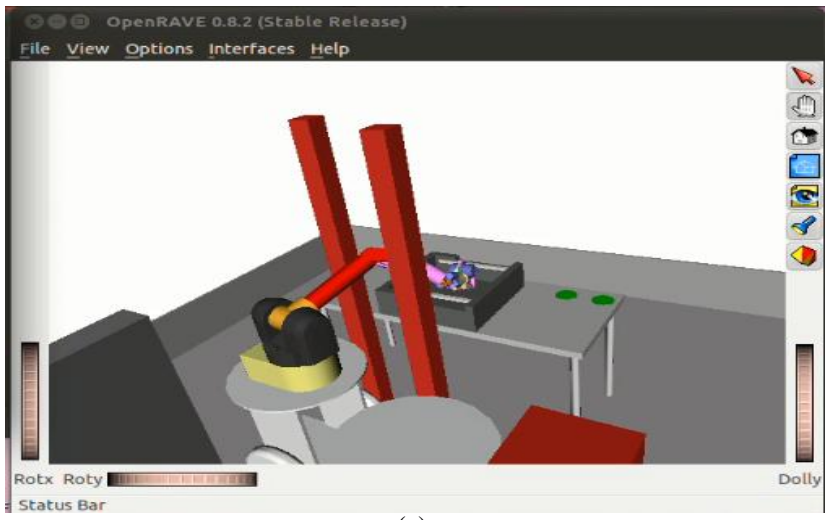

(c)

Fig. 12. Two obstacles between the object and the desired position.

Fig. 12 illustrates the presence of two obstacles between the object and the desired position affects on the time to move the object to the desired position.

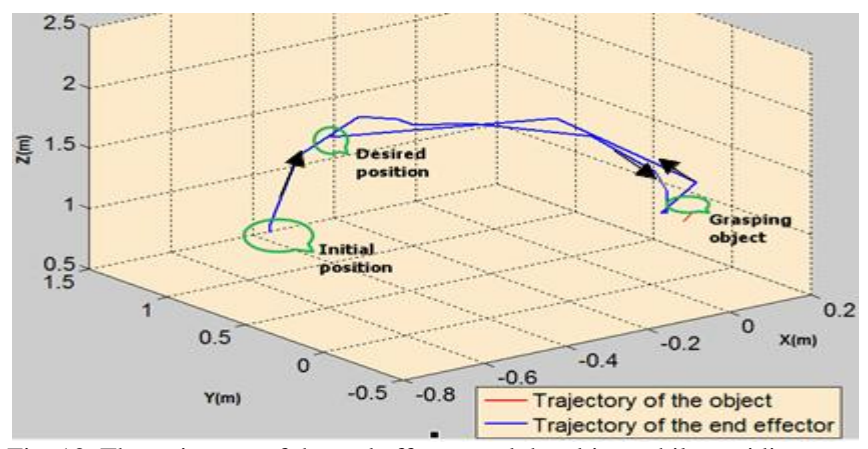

Fig. 13. The trajectory of the end effector and the object while avoiding two obstacles between the object and the desired position. 
Fig. 13(a) illustrates the curves of the second test: the robot grasps the object in time $T_{\text {grasp }}=3.67 \mathrm{~s}$ and $T_{\text {end }}=8.99 \mathrm{~s}$, which moves according to the $\mathrm{Z}$ axis with velocity $V 2$, Fig. 13(b) illustrates the curves of the second test: the robot grasps the object in time $T_{\text {grasp }}=2.93 \mathrm{~s}$ and $T_{\text {end }}=8.83 \mathrm{~s}$, which moves in the plane $(\mathrm{Y}, \mathrm{Z})$ with velocity $V 2$, Fig. 13(c) illustrates the curves of the second test: the robot grasps the object in time $T_{\text {grasp }}=3.12 \mathrm{~s}$ and $T_{\text {end }}=10.62 \mathrm{~s}$, which moves in the space $(\mathrm{X}, \mathrm{Y}, \mathrm{Z})$ with velocity $V 2$.

2) Case study №2: Moving object in the presence of two obstacles between the arm and the object

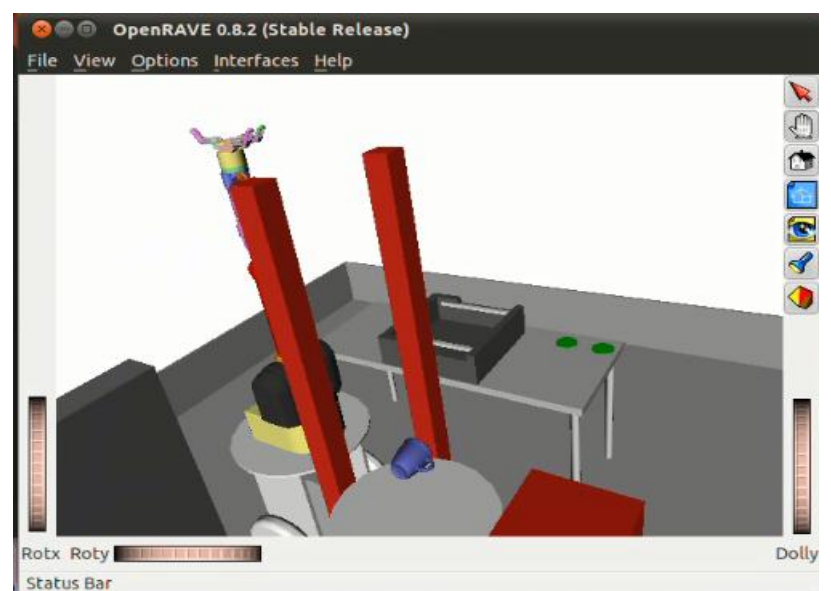

(a)

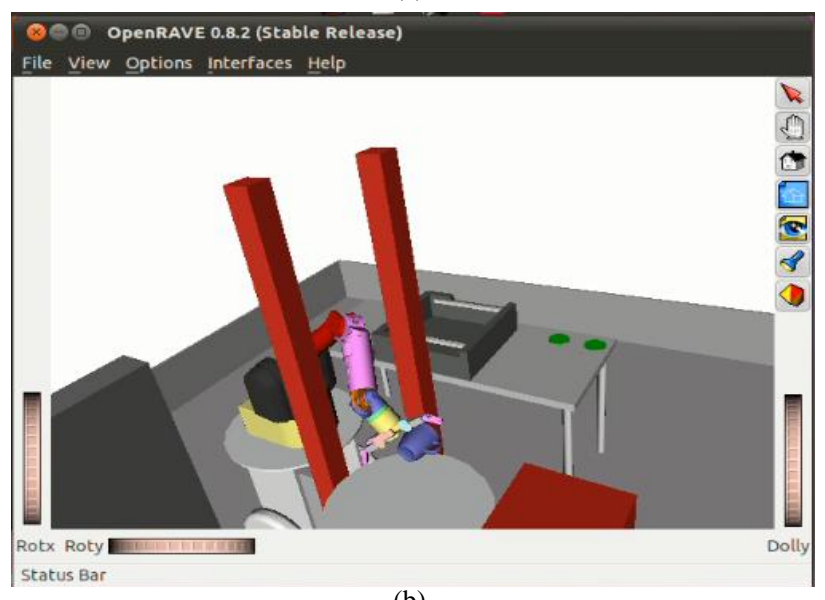

(b)

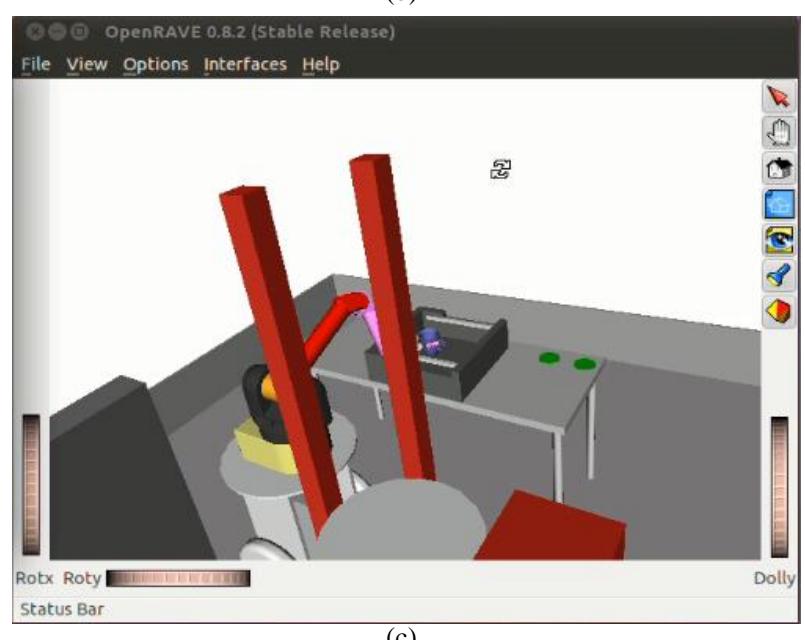

Fig. 14. Two obstacles between the arm and the object.

The conclusion tired from the Fig. 14: the presence of two obstacles between the arm and the object affects on the time to grasp the moving object.

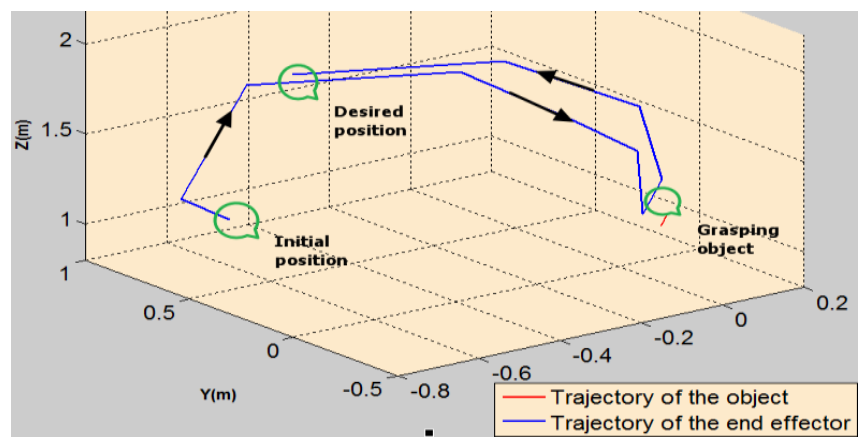

Fig. 15. The trajectory of the end effector and the object while avoiding two obstacles between the arm and the object.

Fig. 15(a) illustrates the curves of the second test: the robot grasps the object in time $T_{\text {grasp }}=3.21 \mathrm{~s}$ and $T_{\text {end }}=7.93 \mathrm{~s}$, which moves according to the $\mathrm{Z}$ axis with velocity $V 2$, Fig. 15(b) illustrates the curves of the second test: the robot grasps the object in time $\mathrm{T}_{\text {grasp }}=3.37 \mathrm{~s}$ and $\mathrm{T}_{\mathrm{end}}=8.98 \mathrm{~s}$, which moves in the plane $(\mathrm{Y}, \mathrm{Z})$ with velocity $V 2$, Fig. 15(c) illustrates the curves of the second test: the robot grasps the object in time $\mathrm{T}_{\text {grasp }}=3.47 \mathrm{~s}$ and $\mathrm{T}_{\text {end }}=7.50 \mathrm{~s}$, which moves in the space $(\mathrm{X}, \mathrm{Y}, \mathrm{Z})$ with velocity $V 2$.

\section{CONCLUSION}

We have presented a simulation to grasp a moving object with different velocities while avoiding obstacles and move this object to a desired position using the 7-DoF robotic arm with the Barret hand by using the RRT algorithm. This algorithm allowed us overcome the problem of the inverse kinematics by exploiting the nature of the Jacobian as a transformation from a configuration space to workspace.

We present results separately of the time for grasping the moving object which moves with different velocity while the obstacles are absent and present and the time to put this object in a desired position. Firstly, the object moves with velocity $V 1$ Second the object moves with velocity $V 2=4 V 1$. The proposed algorithm successfully grasping the moving object in a rational time and put it in a desired position.

Times are nigh in the different test. The presence of obstacles, increasing the speed of grasping the object. The direction of movement of the object affects on the time of grasping the object and on the time to put the object in a desired position. The times recorded in the presence of the obstacle are slightly higher than recorded in the absence of the obstacle.

In this article, we proposed an algorithm for grasping a moving object in the presence of a fixed obstacle. Future work will aim at improving the grasping of a moving object in the presence of a movable obstacles.

\section{REFERENCES}

[1] F. Ruggiero, "Grasp and manipulation of objects with am ulti-fingered hand in unstructured environments," Ph.D. Thesis, Universita Deglistudi Di Napoli, 2010.

[2] T. A. Leper and K. Hsiao, "Strategies for human-in-the-loop robotic grasping," Robot Manipulation and Programming, 2012.

[3] K. Nagase and Y. Aiyana, "Grasp motion planning with redundant dof of grasping pose," Journal of Robotics and Mechatronics, vol. 25, no. 3, 2013. 
[4] H. Faster, "A robot ping pong player: Optimized mechanics, high performance 3 dvision, and intelligent sensor control," Roboter System, pp. 161-170, 1990.

[5] M.-S. Kim, J.-H. Koh, H. Q. P. Nguyen, and H.-J. Kang "Robot visual servo through trajectory estimation of a moving object using kalman filter," in Emerging Intelligent Computing Technology and Applications, D.-S. Huang et al., Eds. Springer Berlin /Heidelberg, 2009, vol. 5754, pp. 1122-1130.

[6] F. Husain, Real Time Tracking and Grasping of a Moving Object from Range Video, 2013.

[7] N. Hushing, "Control of a robotic manipulator to grasp a moving target using vision," in Proc. IEEE Int. Conf. Robotics and Automation, 1990 pp. 604-609.

[8] A. Kiva, "On adaptive vision feed back control of robotic manipulators," in Proc. IEEE Conf. Decision and Control, 1991, pp.1883-1888.

[9] B. M. J. Canny, "Easily computable optimum grasps in2-dand 3-d," in Proc. IEEE International Conference on Robotics and Automation, 1994, pp. 739-747.

[10] N. T. Nomura, "Integrated visual serving system to grasp industrial parts moving on conveyer by controlling 6 dof arm," in Proc. the IEEE International Conference on Systems, Man, and Cybernetics, 2000, pp. 1768-1775.

[11] M. C. D. S. Sen, "Identification and prediction of a moving object using realtime global vision sensing," in Proc. the 20th IEEE Instrumentation and Measurement Technology Conference, 2003, vol. 2, pp. 1402-1406.

[12] J. Z. Ge, "A real time stereo visual servoing for moving object grasping based parallel algorithms," in Proc. IEEE Conference on Industrial Electronics and Applications, 2007, pp. 286-0289.

[13] J. B. Balkenius, "Event prediction and object motion estimation in the development of visual attention," in Proc. the Fifth International Workshop on Epigenetic, 2005.

[14] A.-B. S. Yeoh, "Accurate real-time object tracking with linear prediction method," in Proc. International Conference on Image Processing, 2003, vol. 3.

[15] Z.-K. Matas, "Learning efficient linear predictors for motion estimation," in Proc. 5 Indian Conference on Computer Vision, Graphics and Image Processing, Springer-Verlag, Madurai, India, 2006.

[16] J. Fuentes-Pacheco, "Binocular visual tracking and grasping of a moving object with a 3 dtrajectory predictor," Proceedings of International Journal of Applied Research and Technology, vol. 7, no. 3, 2009.

[17] J.-W. P. S.-J. Kim and J. Lee, "Implementation of tracking and capturing a moving object using a mobile robot," International Journal of Control Automation and Systems, vol. 3, p. 444, 2005.

[18] D. Berenson, Manipulation Planning with Workspace Goal Regions, The Robotics Institute, Carnegie Mellon University, USA, 2009.

[19] J. K. M. Stilman, J.-U. Schamburek, and T. Asfour, "Manipulation planning among movable obstacles," IROS, 2007.

[20] K. K. Y. Hirano and S. Yoshizawa, "Image-based object recognition and dexteroush and/arm motion planning using rrts for grasping in cluttered scene," IROS, 2005.

[21] S. LaValle and J. Kuffner, "Rapidly-exploring random trees: Progress and prospects," WAFR, 2000.

[22] S. M. LaValle. (2006). Planning algorithms. Cambridge, U.K.: Cambridge University Press. [Online]. Available: http://planning.cs.uiuc.edu/
[23] R. D. D. Bertram, J. Kuffner, and T. Asfour, "An integrated approach to inverse kinematics and path planning for redundant manipulators," ICRA, 2006.

[24] E. Drumwright and V. Ng-Thow-Hing, "Toward interactive reaching in static environments for humanoid robots," in Proc. IEEE/RSJ International Conference on Intelligent Robots and Systems, 2006, pp. 846-851.

[25] D. F. M. V. Weghe and S. Srinivasa, "Randomized path planning forredundant manipulators without inverse kinematics," Humanoids, 2007.

[26] S. S. S. D. Berenson, Pittsburgh and by the National Science Foundation under Grant No. EEC-0540865, 2009.

[27] M. V. Weghe, D. Ferguson, and S. S. Srinivasa. Randomized path planning for redundant manipulators without inverse kinematics. [Online]. Available: http://www.ri.cmu.edu/pub_files/pub4/vande_weghe_michael_2007_ 1/vande_weghe_michael_2007_1.pdf

[28] E. L. Damian, "Grasp planning for object manipulation by an autonomous robot," Master's thesis, National Institute of Applied Sciences of Toulouze, 2006

[29] M. W. Spong and M. Vidyasagar, Robot Dynamics and Control, 1989.

[30] WAM Arm User's Guide. [Online]. Available: www.barrett.com

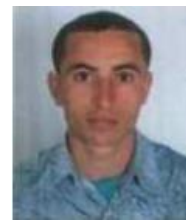

Ali Chaabani was born in Kairouan, Tunisia in 1987. He received the principal engineering diploma in compute science in 2011 from the Sfax National School of Engineering (ENIS), the master degree of computer science and automation in 2013 from the National Institute of Applied Sciences and Technology (INSAT).

Now, he is a PhD student in Tunis National School of Engineering (ENIT) and a researcher in Informatics Laboratory for Industrial Systems (LISI) at the National Institute of Applied Sciences and Technology (INSAT).

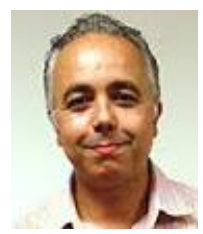

Mohamed Sahbi Bellamine is an assistant professor in the National Institute of Applied Sciences and Technology, University of Carthage. Now, $\mathrm{He}$ is a member of Laboratory of Computer Science of Industrials Systems (LISI) at the National Institute of Applied Sciences and Technology (INSAT). His domain of interests is human-robot interaction, social \& sociable robots.

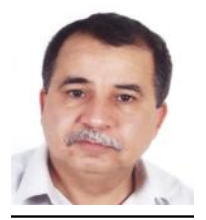

Moncef Gasmi was born in Tunis, Tunisia in 1958. He received the principal engineering diploma in electrica engineering in 1984 from the Tunis National School of Engineering (ENIT), the master degree of systems analysis and computational treatment in 1985, the doctorate in automatic control in 1989 and the state doctorate in electrical engineering in 2001.

Now, he is a professor and director of the Informatics Laboratory for Industrial Systems (LISI) at the National Institute of Applied Sciences and Technology (INSAT). His domain of interests is related to the modeling, analysis and control of complex systems. 